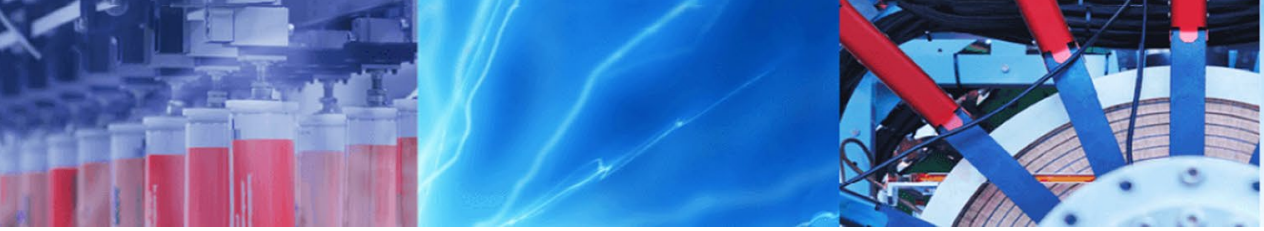

Research Article

\title{
Inertial gravity currents over a permeable bottom in trapezoidal type cross-section channels: shallow-water and box-model solutions
}

\author{
T. Zemach ${ }^{1}$
}

Received: 14 May 2020 / Accepted: 27 August 2020 / Published online: 10 September 2020

(c) Springer Nature Switzerland AG 2020

\begin{abstract}
We consider an inertial gravity current spreading over a horizontal permeable floor in channels of trapezoidal type cross-section (CS). A closed one-layer shallow-water Boussinesq inviscid formulation is presented. It is shown that initially the porosity has little influence on the spreading of the current, but it decreases the distance of propagation during the following stages of propagation. The position of the porosive boundary is also examined. Qualitatively, the behaviour of the current is similar to one spreading over a fully-permeable bottom, however the current is faster as the porosive domain starts far from the origin. We illustrate the methodology for flows in typical trapezoidal type CS channels $f(z)=1 / 2\left(1+z^{\alpha}\right)$ (here the constant $\alpha$ is positive) and show that the slowest propagation is achived in containers of rectangular form $(\alpha=0)$.
\end{abstract}

Keywords Gravity currents · Permeable surface $\cdot$ Non-rectangular cross-section

\section{Introduction}

A gravity current (GC) is formed when fluid of one density $\rho_{1}$ propagates mainly, in the horizontal direction into a fluid of another density $\rho_{2}\left(\rho_{1}>\rho_{2}\right)$. In nature, many currents flow over an impermeable media (Simpson [15]). This includes turbidity GCs, avalanches and oceanic fronts ([5, 16]). However, in many cases of interest, the GC encounters a topography or spread over porous media. Examples include accidental collapse of storage tanks, containing dense gases, surrounded by gravel beds.

These problems have received attention in previous studies which were focused on the GCs flow propagating over an permeable bottom in a rectangular channel. Thomas et al. [18] and Acton et al. [1] performed laboratory experiments for a thin layer of porous substrate. Ungarish and Huppert [21] formulated a shallow-water (SW) model which was able to predict three main current's spreading inertial phases and were consistent with the previously published experimental outcomes : initially the propagation of the current is similar to the classical case with impermeable bottom, but in contrast, the height of the front does not remain constant and decreases systematically; afterwards, the porosity slows-down the current and shortens the effective distance of propagation. This is a transition stage to the third similarity stage of propagation. The third stage has recently received attention by Zemach and Ungarish [23] and useful insights about the influence of the porous boundary, as compared with the classical similarity behavior, were noted: the distance of propagation is shorter, the profile of the velocity is deflected to lower values at the nose, and the height of the current is reduced mostly at the tail. Viscous gravity currents also were widely discussed $([4,8,13,17])$.

However, all previous studies were restricted to gravity currents spreading in a rectangular channel, while GCs generated and propagating in channels with non-rectangular or, specifically, trapezoidal-type cross-sections (CS) are practical configurations in nature ([7, 9-11, 22]). The examples of such currents include GCs propagating in

T. Zemach, tamar.zemach@yahoo.com | 'Department of Computer Science, Tel-Hai College, Tel-Hai, Israel. 
submarine channels, valleys and rivers for which the floor and/or the sides are usually porous and therefore part of the fluid is absorbed in the soil. The objective of this paper is to close this gap of knowledge.

The structure of the paper is as follows. In Section 2, we formulate the SW model of motion. Some numerical solutions of SW are presented and discussed in Sect. 3. Simplified box-model is discussed in Sect. 4. Finally, in Sect. 5 some concluding remarks are given.

\section{Formulation}

We consider a layer of fluid of density $\rho_{1}$ and kinematic viscosity $\nu$ which propagates over a permeable horizontal surface at $z=0$ into an ambient fluid of constant density $\rho_{2}$ in a container of a uniform (in the $x$-direction) CS shape of trapezoidal type. As depicted in Figs. 1 and 2, the bottom and the top of the half-infinite container are at $z=0$ and $z=H$ and its side-walls are given by $y=-f_{1}(z)$ and $y=f_{2}(z)$ functions. The flow depends actually on the width function, $f(z)=f_{1}(z)+f_{2}(z)$, which is assumed continuous and positive. We restrict the model to trapezoidal-type function of the form $f(z)=0.5+0.5 z^{\alpha}$. Here $\alpha$ is non-negative constant. The current is driven by the reduced gravity $g^{\prime}$ defined by

$g^{\prime}=\frac{\rho_{1}-\rho_{2}}{\rho_{2}} g$,

where $g$ is the acceleration due to gravity. By using the Boussinesq approximation we consider the values of the densities $\rho_{1} / \rho_{2} \approx 1+$.

We assume that the width of the channel is much larger than the thickness of the current, and hence the flow can be treated as two-dimensional in a $x-z$ coordinates

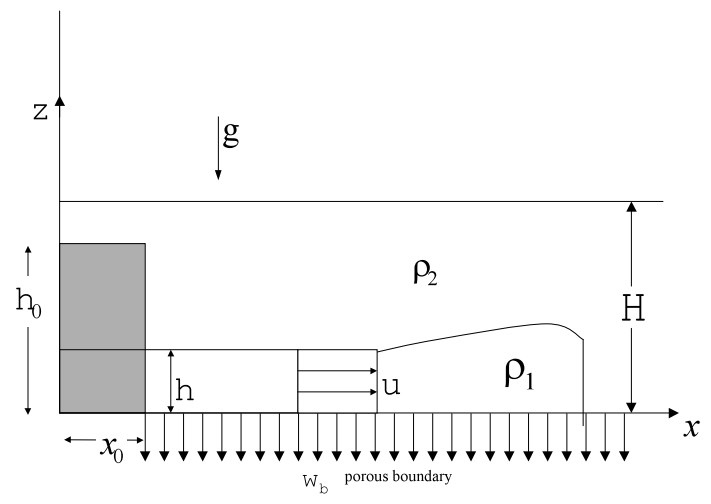

Fig. 1 Side-view of the system: the height of the ambient fluid is $H$; Initially the current is located behind a lock of height $h_{0}$ and length $x_{0}$. The propagation to $x$ direction

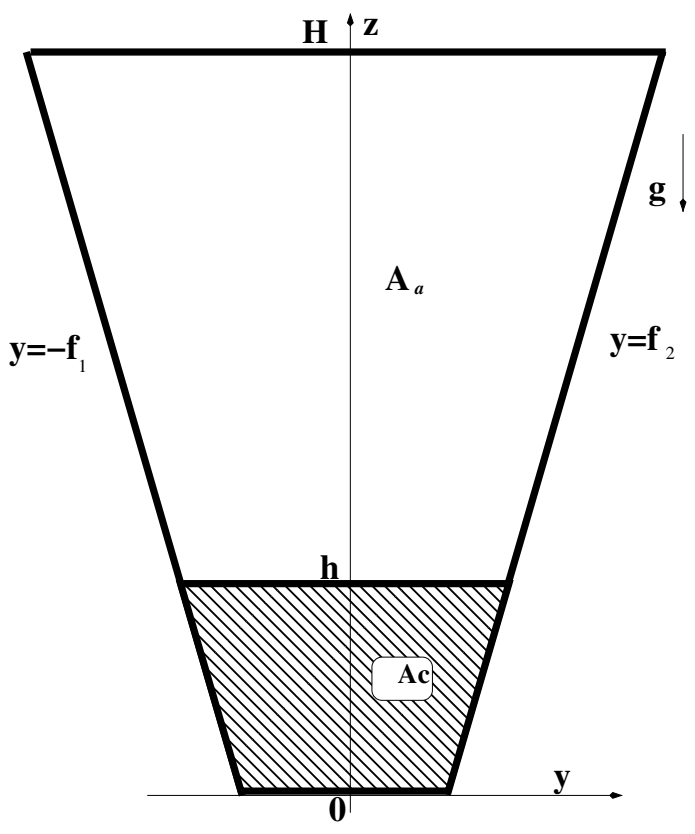

Fig. 2 Cross-section of channel. Here the width of the container is given by $f(z)=f_{1}(z)+f_{2}(z)$

system with corresponding $\{u, w\}$ velocity components. The propagating fluid originally, at time $t=0$, is at rest in channel of length $x_{0}$ and height $h_{0}$. We assume that in the ambient fluid domain, the velocity of the flow is zero.

The porous surface contributes the total flux term to the right hand side of the continuity equation:

$Q_{b}=-\frac{1}{\tau} h f(0)=-\frac{1}{\tau} \frac{h}{2}$,

where the time-constant $\tau$ can be measured in the laboratory with modest efforts (Thomas et al. [18]).

We note by $h(x, t)$ the height of the current (initially, $\left.h=h_{0}\right)$ and by $u(x, t)$ its velocity, averaged over the area of the current (initially $u=0$ ). We assume a shallow current with $h_{0} \ll x_{0}$.

It is convenient to use dimensionless variables. Here, we denote the dimensional variables by asterisks, and transform to the dimensionless counterpart (with no special notation) as follows:

$$
\begin{aligned}
& \left\{x^{*}, y^{*}, z^{*}, h^{*}, H^{*}, t^{*}, u^{*}\right\} \\
& \quad=\left\{x_{0} x, h_{0} y, h_{0} z, h_{0} h, h_{0} H, T_{\text {ref }} t, U_{\text {ref }} u\right\},
\end{aligned}
$$

where $U_{\text {ref }}=\sqrt{h_{0} g^{\prime}}$ and $T_{\text {ref }}=\frac{x_{0}}{U_{\text {ref }}}$. We introduce additional non-dimensional scaling parameter (constant)

$\lambda=\frac{T_{r e f}}{\tau}$

\section{SN Applied Sciences}


which reflects the ratio between the typical time of propagation of the nose to the typical time of descent of the interface due to the porosity of the boundary, the former over a length $x_{0}$ and the latter over a height $h_{0}$. Let the value of $\lambda$ be a constant in the porous domain. As the current spreads out, the height and the volume of the current decrease, but the area of absorption increases.

The Reynolds number of the horizontal flow is assumed to be large and is defined by $R e=\frac{h_{N} u_{N}}{v}$, where the subscript $N$ denotes value associated with the nose of the current.

\subsection{The governing equations}

Let $A_{C}$ and $A_{T}$ denote the total cross section of the current and the total of the channel:

$$
\begin{gathered}
A_{c}=A(h)=\int_{0}^{h} f(z) d z=\frac{1}{2} h+\frac{1}{2} \cdot \frac{h^{\alpha+1}}{\alpha+1}, \\
A_{T}=\int_{0}^{H} f(z) d z=\frac{1}{2} H\left(1+\frac{H^{\alpha}}{\alpha+1}\right) .
\end{gathered}
$$

There are two equations of motion-the continuity equation and the momentum equation:

$\left(\begin{array}{l}h_{t} \\ u_{t}\end{array}\right)+\left(\begin{array}{cc}u & \Psi(h) \\ 1 & u\end{array}\right)\left(\begin{array}{l}h_{x} \\ u_{x}\end{array}\right)=\left(\begin{array}{c}G(h) \\ 0\end{array}\right)$.

Here

$\Psi(h)=\frac{A(h)}{f(h)}=\frac{h\left(\alpha+1+h^{\alpha}\right)}{(\alpha+1)\left(1+h^{\alpha}\right)}$

and

$G(h)=-\lambda \frac{h}{1+h^{\alpha}}$.

We note that the continuity equation of (6) can also be written in the conservation form by

$\frac{\partial A}{\partial t}+\frac{\partial}{\partial x}(u A)=-\frac{1}{2} \lambda h$.

In many realistic situations the bottom boundary can be porosive starting at the beginning of the container (at $x=0$ ) or, alternatively, to be non-porous below the lock of the reservoir and to become porosive only after that (at $x=1)$. It is important to keep in mind that if there is a non-porous domain in the boundary, then the value of $\lambda$ should be set to zero for the impermeable region in the equations of motion.

The system (6) is hyperbolic with characteristic relationships given by

$d h \pm \sqrt{\Psi(h)} d u=G(h) d t$ on $\frac{d x}{d t}=u \pm \sqrt{\Psi(h)}$.

The initial conditions are $u=0, h=1$ at $t=0$ in the lock $0 \leq x \leq 1$. The corresponding boundary conditions (in dimensionless form ) are $u=0$ at the backwall $x=0$ and

$u_{N}=h_{N}^{1 / 2} \operatorname{Fr}\left(\frac{h_{N}}{H}\right)$

at the nose $x=x_{N}$. Here $F r$ is Froude number function given by (see $[2,6,20])$ :

$\operatorname{Fr}\left(\frac{h_{N}}{H}\right)=\operatorname{Fr}(\varphi)=\left[\frac{2(1-\varphi)^{2}}{1+\varphi}(1+K)\right]^{1 / 2}$,

where

$\varphi=\frac{A_{c}}{A_{T}} \quad$ and $\quad K=\frac{\int_{0}^{h} z f(z) d z}{h \cdot\left[A_{T}-A_{c}\right]}$.

\section{Discussion}

To solve the model, we need an efficient method for obtaining the height $h(x, t)$, velocity $u(x, t)$, and distance of propagation $x_{N}(t)$ from our formulation. In general, the system of equations (6) with realistic initial/boundary conditions, cannot be solved analytically. We use an approach which was previously successfully used $([14,21$, 22]) and first map the $x$-coordinate to $\eta=x / x_{N}(t)$ (so the transformed domain of solution is $[0,1])$ and then apply a finite-difference two-step Lax-Wendroff method ( Bonnecaze et al. [3], Morton and Mayers [12]). Typical results of SW, displayed below, were obtained with 200 grid points in the $\eta \in[0,1]$ with time step of 0.001 .

The typical behavior of the time-dependent currents propagating in containers of trapezoidal CS
Fig. 3 SW model results for trapezoidal configuration with a permeable floor $(x \geq 0)$ : profiles of $h$ and $u$ as functions of $x$ at various $t=1,2,5,7,10,15,20,30$. Here $f(z)=0.5+0.5 z, H=10, \lambda=0.1$
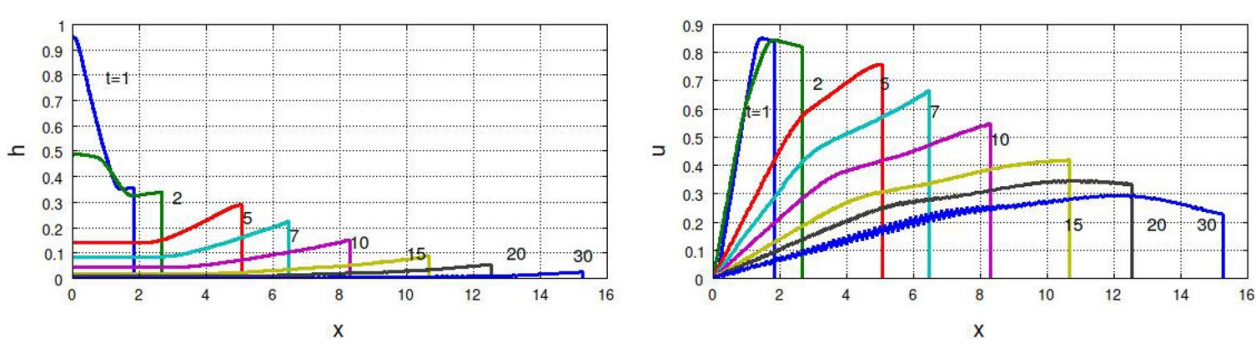

SN Applied Sciences A SPRINGER NATURE journa 
$(f(z)=0.5+0.5 z)$ with fully-permeable floor $(x \geq 0)$ with $H=10$ and $\lambda=0.1$ is displayed in Fig. 3 .

Initially, the height of the nose decreases from its initial value $\left(h_{N}(0)=1\right)$ to $h_{N} \approx 0.34$. The porosity of the floor causes a continuous decrease of $h_{N}$, as expected. The next stage of propagation is characterized by the decreasing height of the nose and speed. This stage is a transient and it finishes at $t \approx 15$. Finally, the current approaches a similarity solution with typical head-up tail-down smooth profile of $h(x, t)$ and non-monotonic function of $x$ for the velocity $u(x, t)$.

Figure 4 shows the same configuration as in Fig. 3 in container with permeable floor starting at $x=1$. Qualitatively, the behavior of the current is similar to that obtained for a fully-permeable floor case shown above. However, the current is faster. Also, the height and the volume of the current are kept higher than in the previous case.

To check if and how the shape of the container affects the distance of propagation of the flow, we show in Fig. 5 the distances of propagation $x_{N}$ versus $t$ for four different types of trapezoidal-type containers with $f(z)=0.5+0.5 z^{\alpha}$ and $\alpha=0,0.5,1.0,2.0$. The height $H$ of the container and value of porous parameter $\lambda$ for these graphs were identical $(H=10, \lambda=0.1)$.

We recall that the $\alpha=0$ solution coincides with the classical rectangular CS case (the relevant results were presented by Ungarish and Huppert [21]). Initially, the current propagation is not effected by the form of the container (we can see that for $t \leq 5$ the all lines coincide). Then, in the rectangular container the current decreases its speed dramatically, in compare with other channels. The differences between other containers are insignificant till $t \approx 15$ and become more significant (in about 6\%) in more progressive times. The interesting conclusion here is that the slowest propagation of the current is observed in the rectangular container $(\alpha=0)$ with decreasing of about $17 \%$ in the distance $x_{N}$ at advanced times.

We can explain this as a combination of two effects. (1) It is evident that the value of $\alpha$ is a measure of the rate of expansion of the ambient in the $z$ direction, as seen from the current. Therefore a larger $\alpha$, for a fixed $H$, means that the current moves in a larger ambient, produces a smaller return-flow, and hence can develop a larger $u_{N}$; this was detected in [22]. (2) For a current of given volume, the area of contact with the porous bottom decreases as a increases. Combining the two effects, we conclude that the current in the rectangular $\alpha=0$ channel encounters a larger return flow and is subject to a larger bottom drainage, therefore propagates more slowly than the $\alpha>0$ counterpart.

Figure $5 b$ ) shows the decreasing of the volume of the current in compare to its initial value. As we can see, as $V(t) / V(0)$ becomes greater, the current becomes faster.

We note that the presence of the porous layer changes the propagation trends in compare with the channels with impermeable bottoms (here we use results obtained for the impermeable floor discussed in [22]). In Fig. 6 the distance of propagation as a function of $t$ in impermeable bottom containers $(\lambda=0)$ is shown for comparison. As we can see, the slowest current is still the one spreading in the rectangular container (with $\alpha=0$ ). However, propagation in other channels differs only slightly from each other. The trends are also different : the linear $\alpha=1$ is the fastest one and the propagation in parabolic container with $\alpha=2$, faster than in one with $\alpha=0.5$.
Fig. 4 The porous floor starts at $x>1$ and impermeable floor for $x \leq 1$. Other parameters are as in Fig. 3
Fig. 5 Distance of propagation of the current $x_{N}$ and volume ratio $V(t) / V(0)$ as functions of $t$ in containers with permeable floor starting at $x=0$. Here $H=10, \lambda=0.1$. $f(z)=0.5\left(1+z^{\alpha}\right), \alpha=0,0.5,1,2$
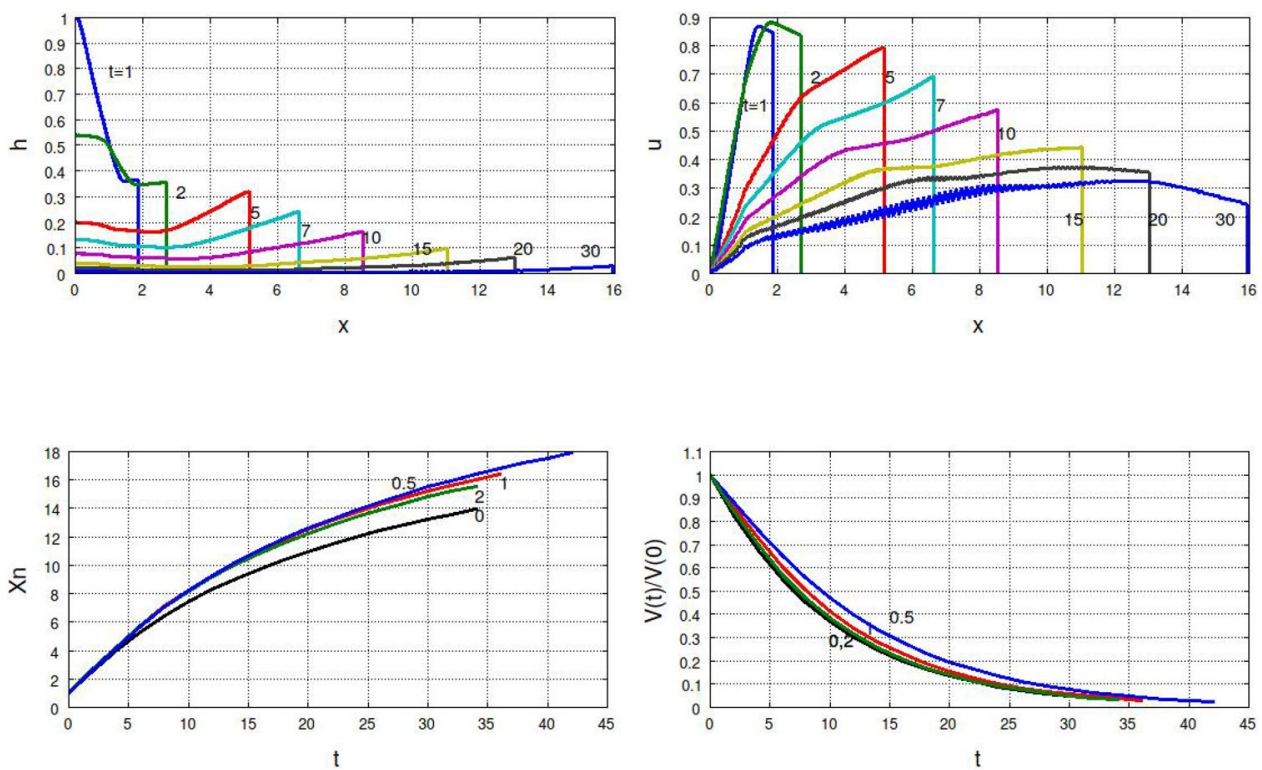


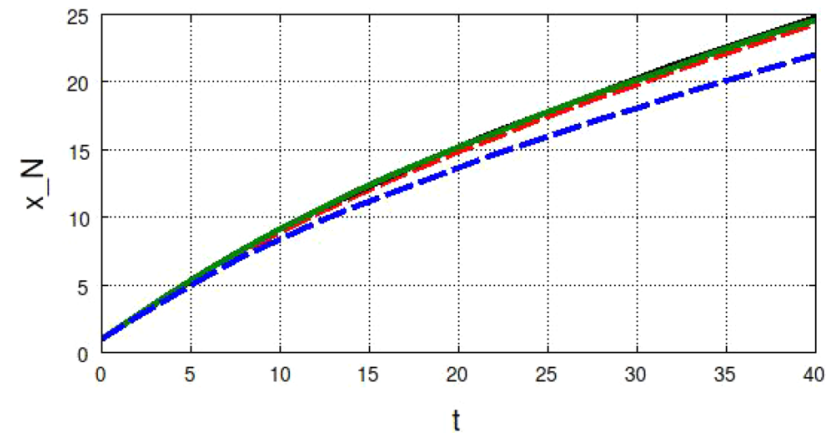

Fig. 6 Distance of propagation of the current $x_{N}$ as function of $t$ in the containers with full impermeable floor $(\lambda=0)$. Here $H=10, f(z)=0.5\left(1+z^{\alpha}\right): \alpha=0$ (blue), 0.5 (red), 1.0 (black), 2.0 (green)

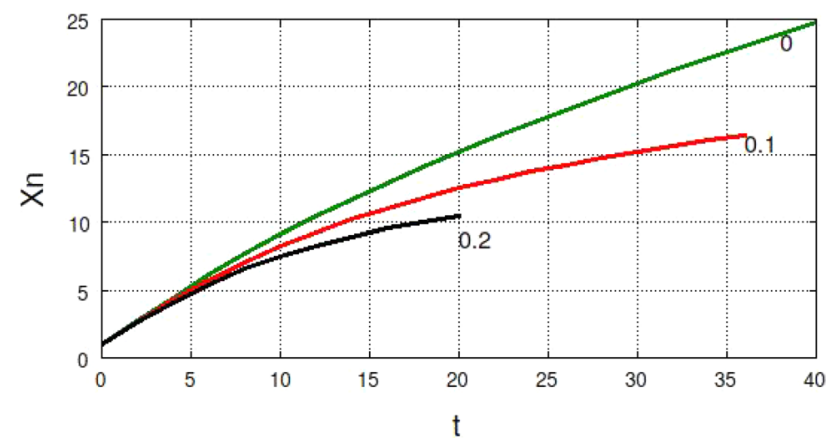

Fig. 7 Distance of propagation of the current $x_{N}$ as function of $t$ in the trapezoidal container with permeable floor starting at $x=0$. Here $H=10, f(z)=0.5(1+z)$ and various values of $\lambda=0,0.1,0.2$

Figure 7 gives a quick insight into the influence of the parameter $\lambda$ on the propagation distance in containers of trapezoidal CS $(f(z)=0.5+0.5 z)$ (other geometries also have been examined). Initially, the current "does not feel" the permeable floor and propagates till $t \approx 5$ as one over an impermeable boundary. (This behavior is consistent with the results shown for early times in Fig. 6).

Afterwards, the porosity slows-down the current and shortens the effective distance of propagation. These effects become more pronounced as the values of parameter $\lambda$ increases. For example, for $\lambda=0.1$, the permeability of the floor causes dramatic decreasing of $\approx 50 \%$ at the advanced times in distance of propagation $x_{N}$ in compare to currents which propagate in channels with impermeable surfaces.

Figure 8 shows the behavior of the volume ratio $V(t) / V(0)$ for trapezoidal CS $(f(z)=0.5+0.5 z)$ for fully permeable $(x>0)$ and partially permeable $(x>1)$ bottoms. The $V(t) / V(0)$ decreases more pronounced as

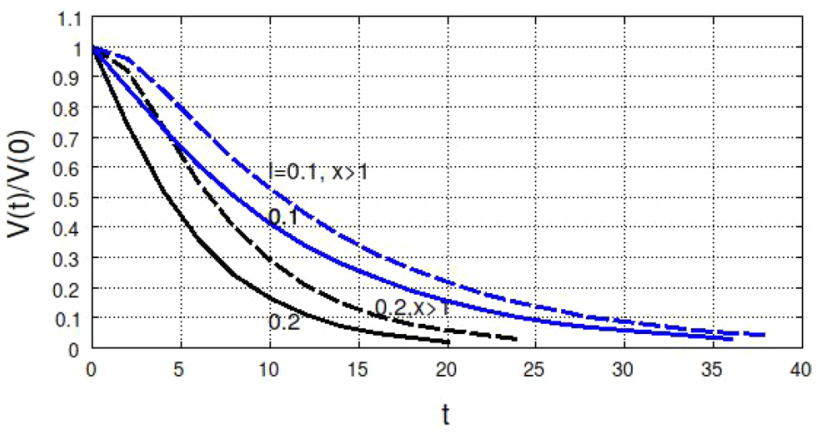

Fig. 8 Volume ratio $V(t) / V(0)$ as function of $t$ in the trapezoidal container with permeable floor starting at $x=0$ (solid line) and at $x=1$ (dashed line). Here $H=10, f(z)=0.5(1+z)$ and various values of $\lambda=0.1,0.2$

$\lambda$ increases. As expected, for $x>1$ cases, the $V(t) / V(0)$ decreasing less dramatically than for the fully-porosive case.

\section{Box-model}

The "box-model" is an approach that views the current as a control volume of uniform area $A(h(t))$ and length $x_{N}(t)$, whose total volume is not constant $V$.

The current is assumed to be a "box" whose volume is

$V(t)=\int_{0}^{x_{N}} A(h) d x$

Derivation of (14) yields:

$\frac{d V}{d t}=\int_{0}^{x_{N}} \frac{d A(h(t))}{d t} d x+A(h(t)) \cdot \frac{d x_{N}}{d t}$.

Next, substitution of (15) into the horizontally-integrated expression for the conservation of volume (9) produces the following equation:

$\frac{d V}{d t}=-\frac{1}{2} \lambda h \cdot x_{N}$

which can be rewritten by

$\frac{d}{d t}\left(0.5 h_{N}\left(1+\frac{1}{\alpha+1} h_{N}^{\alpha}\right) \cdot x_{N}\right)=-\frac{1}{2} \lambda h \cdot x_{N}$.

The nose condition (11) is

$\frac{d x_{N}}{d t}=F r \cdot h_{N}^{1 / 2}$.

We note that (17)-(18) is the system of ODEs for the length $h_{N}(t)$ and distance $x_{N}(t)$ which can be solved numerically. 


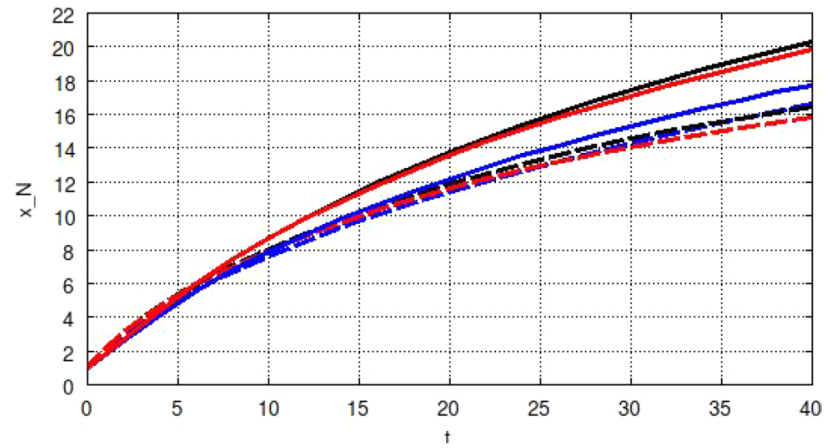

Fig. 9 SW(solid line) and box-model(dashed line) calculations for propagation as function of $t$ in trapezoidal containers with $f(z)=0.5+0.5 z^{\alpha}: \alpha=0$ (blue); $\alpha=1$ (black) and $\alpha=2$ (red). Here $H=10$ (deep stage) and $\lambda=0.05$. The bottom is fully permeable $(x>0)$

Typical solutions of (17)-(18) are shown in Fig. 9 for $\alpha=0,1,2$ and $\lambda=0.05$ in containers with fully-permeable $(x \geq 0)$ floor. Here $H=10$. Results obtained from SW runs also shown. Initially $(t \leq 7)$ the agreement is excellent, while both the SW and the box-model solutions differ only slightly from each other independently of the value of $\alpha$. Later, the box-model approximation predicts a slower propagation of the current than the SW equations with divergence of about $15 \%$ between the models.

\section{Summary}

We presented a theoretical Boussinesq one-layer SW model which predicts the shape $h(x, t)$, the averaged velocity $u(x, t)$ and the distance of propagation $x_{N}(t)$ for gravity current flows spreading over a permeable surface in channels of trapezoidal-type $f(z)=0.5\left(1+z^{\alpha}\right)$ (where $\alpha$ is nonnegative constant; $f(z)$ is scaled by $f\left(h_{0}\right)$ and $z$ by $\left.h_{0}\right)$. The effect of the porosity is described by means of a parameter $\lambda$ which is assumed to be small. The SW model limitations have been presented widely in the literature ([19]) and they are relevant also here. However, the big advantage of these models is their simplicity and the ability to gain insights concerning the main stages of propagation of the current. In general, the porosity slows-down the current and shortens the effective distance of propagation in all examined geometric configurations. These effects become more pronounced as the value of parameter $\lambda$ increases. Comparison between partially-permeable and fully-permeable bottoms shows that qualitatively, the behavior of the current is similar in both configurations, however, in the former the current is faster and its volume decreases more moderately.

For a given value of $\lambda$, initially, the current propagation is not effected by the form of the container, but afterwards the speed of the current decreases much more rapidly in the containers of rectangular CS than in channels of trapezoidal types. As a result, the slowest propagation of the current was observed in the rectangular container $(\alpha=0)$.

We hope that the present work will motivate further work on this problem. In particular, experimental or Navier-Stokes simulations with which the model presented here can be compared. Additional interesting extensions include presence of an external source in a system and expanding the model to channels of general cross-sections.

\section{Compliance with ethical standards}

Conflicts of interest The author declares that she has no conflict of interest.

\section{References}

1. Acton J, Huppert $\mathrm{H}$, Worster $\mathrm{M}$ (2001) Two-dimensional viscous gravity currents flowing over a deep porous medium. J Fluid Mech 440:359-380

2. Benjamin T (1968) Gravity currents and related phenomena. J Fluid Mech 31:209-248

3. Bonnecaze RT, Huppert HE, Lister JR (1993) Particle-driven gravity currents. J Fluid Mech 250:339-369

4. Hatcher L, Hogg AJ, Woods AW (2000) The effects of drag on turbulent gravity currents. J Fluid Mech 416:297-314

5. Hoult D (1972) Oil spreading on the see. Annu Rev Fluid Mech 2:341-368

6. Huppert $H$, Simpson J (1980) The slumping of gravity currents. J Fluid Mech 99:785-799

7. Keramaris E, Prinos P (2016) Gravity currents in a vegetated valley of trapezoidal shape. J Appl Fluid Mech 9-3:1051-1056

8. Lauriola I, Felisa J, Petrolo D, Di Federico V, Longo S (2018) Porous gravity currents: axisymmetric propagation in horizontally graded medium and a review of similarity solutions. Adv Water Resour 115:136-150

9. Marino B, Thomas L (2009) Front condition for gravity currents in channels of non rectangular symmetric cross-section shapes. J Fluids Eng 131:1201-1206

10. Marino B, Thomas L (2011) 2011 dam-break release of a gravity current in a power-law channel section. J Phys: Conf Ser 296:012008

11. Mériaux CA, Kurz-Besson CB (2017) A study of gravity currents carrying polydisperse particles along a V-shaped valley. Eur J Mech B/Fluids 63:52-65

12. Morton KW, Mayers DF (1998) Numerical solution of PDE. Cambridge University Press, Cambridge

13. Pritchard D (2007) Gravity currents over fractured substrates in a porous medium. J Fluid Mech 584:415-431

14. Rottman J, Simpson J (1983) Gravity currents produced by instantaneous release of a heavy fluid in a rectangular channel. J Fluid Mech 135:95-110

15. Simpson J (1997) Gravity currents in the environment and the laboratory. Cambridge University Press, Cambridge

16. Sparks R, Bursik M, Carey S, Gilbert J, Glase L, Sigurdsson H, Woods A (1997) Volcanic Plumes. Wiley, Hoboken 
17. Tanino Y, Nepf HM, Kulis PS (2005) Gravity currents in aquatic canopies. Water Resour Res 41(12):W1240219

18. Thomas L, Marino B, Linden P (1998) Gravity currents over porous substrate. J Fluid Mech 366:239-258

19. Ungarish $M$ (2009) An introduction to gravity currents and intrusions. CRC Press, Boca Raton

20. Ungarish M (2012) A general solution of Benjamin-type gravity current in a channel of non-rectangular cross-section. Environ Fluid Mech 12(3):251-263

21. Ungarish $M$, Huppert $H$ (2000) High-Reynolds-number gravity currents over a porous boundary: shallow-water solutions and box-model approximations. J Fluid Mech 418:1-23

22. Zemach T, Ungarish M (2013) Gravity currents in non-rectangular cross-section channels: analytical and numerical solutions of the one-layer shallow-water model for high Reynolds-number propagation. Phys. Fluids 25(2):026601

23. Zemach T, Ungarish M (2020) Inertial gravity current in rectangular channels over a porous bottom: asymptotic solutions. Eur J Mech/ B Fluids 84:122-128

Publisher's Note Springer Nature remains neutral with regard to jurisdictional claims in published maps and institutional affiliations. 